\title{
Preweaning cost of bovine respiratory disease (BRD) and cost-benefit of implementation of preventative measures in calves on California dairies: The BRD 10K study
}

\author{
S. A. Dubrovsky, ${ }^{1,2}$ A. L. Van Eenennaam, ${ }^{2}{ }^{\oplus}$ S. S. Aly, ${ }^{3,4 *}$ B B. M. Karle, ${ }^{5}$ Paul V. Rossitto, ${ }^{3}$ M. W. Overton, ${ }^{6}$ \\ T. W. Lehenbauer, ${ }^{3,4} \odot$ and J. G. Fadel ${ }^{2 *} \oplus$ \\ ${ }^{1}$ Veterinary Medicine Teaching and Research Center, School of Veterinary Medicine, University of California-Davis, Tulare 93274 \\ ${ }^{2}$ Department of Animal Science, University of California-Davis, Davis 95616 \\ ${ }^{3}$ Veterinary Medicine Teaching and Research Center, School of Veterinary Medicine, University of California-Davis, Tulare 93274 \\ ${ }^{4}$ Department of Population Health and Reproduction, School of Veterinary Medicine, University of California-Davis, Davis 95616 \\ ${ }^{5}$ California Cooperative Extension, Division of Agriculture and Natural Resources, University of California, Orland 95963 \\ ${ }^{6}$ Elanco Animal Health, 2500 Innovation Way, Greenfield, IN 46140
}

\begin{abstract}
Bovine respiratory disease $(\mathrm{BRD})$ is a multifactorial disease that is estimated to affect $22 \%$ of preweaned dairy calves in the United States and is a leading cause of preweaning mortality in dairy calves. Overall cost of calfhood BRD is reflected in both the immediate cost of treating the disease as well as lifetime decrease in production and increased likelihood of affected cattle leaving the herd before their second calving. The goal of this paper was to develop an estimate of the cost of BRD based on longitudinal treatment data from a study of BRD with a cohort of 11,470 preweaned dairy calves in California. Additionally, a cost-benefit analysis was performed for 2 different preventative measures for BRD, an increase of $0.47 \mathrm{~L}$ of milk per day for all calves or vaccination of all dams with a modified live BRD vaccine, using differing assumptions about birth rate and number of calves raised per year. Average short-term cost of BRD per affected calf was $\$ 42.15$, including the use of anti-inflammatory medications in the treatment protocols across all management conditions. The cost of treating BRD in calves appears to have increased in recent years and is greater than costs presented in previous studies. A cost-benefit analysis examined different herd scenarios for a range of cumulative incidences of BRD from 3 to $25 \%$. Increasing milk fed was financially beneficial in all scenarios above a $3 \%$ cumulative incidence of BRD. Use of a modified live vaccine in dams during pregnancy, examining only its value as a form of BRD prevention in the calves raised on the farm, was financially beneficial only if the cumulative incidence of BRD exceeded 10 to $15 \%$
\end{abstract}

Received August 6, 2018.

Accepted September 16, 2019.

*Corresponding authors: saly@ucdavis.edu and jgfadel@ucdavis.edu depending on the herd size and whether the dairy farm was raising any bull calves. The cost-benefit analysis, under the conditions studied, suggests that producers with high rates of BRD may benefit financially from implementing preventative measures, whereas these preventative measures may not be cost effective to implement on dairy farms with very low cumulative incidences of BRD. The long-term costs of calfhood $\mathrm{BRD}$ on lifetime productivity were not factored into these calculations, and the reduction in disease may be associated with additional cost savings and an improvement in calf welfare and herd life.

Key words: bovine respiratory disease, economics, preventative measure, cost-benefit

\section{INTRODUCTION}

Bovine respiratory disease (BRD) is a multifactorial disease that can be caused by both bacteria and viruses. The disease is one of the leading causes of morbidity and mortality in preweaned dairy calves (USDA, 2010, 2012). A recent study of California dairy calves found that around $22.8 \%$ of calves are diagnosed and treated for BRD before weaning and that $19 \%$ of all preweaned deaths can be attributed to BRD (Dubrovsky et al., 2019a). These numbers reflect the high incidence and mortality of this complex disease.

Two previous studies have sought to model and identify costs associated with treating dairy calves with BRD. One of these studies used a computer-based model to estimate farm-specific losses, and not individual calf losses (van der Fels-Klerx et al., 2001). This model was developed using data from Holstein dairy farms in the Netherlands, limiting its usefulness for large US dairy farms. An older model was developed from data collected through the National Animal Health Monitoring System (NAHMS) and is more relevant to 
dairy farms in the United States (Kaneene and Hurd, 1990). However, the latter authors noted that their cost estimations were incomplete and should be used with caution. Studies show that even when calves are successfully treated for BRD, they may never recover sufficiently to be as productive and healthy as their herdmates (Stanton et al., 2012; Schaffer et al., 2016).

The most effective way to decrease costs associated with BRD is to prevent BRD from occurring in the first place (van der Fels-Klerx et al., 2001). In this study, we evaluated both the economic costs of treating BRD in calves, and the short-term (preweaning) cost-benefit of preventative measures to reduce the cumulative incidence of BRD. A model of the cost of calfhood BRD was generated from treatment data collected in a previous study and combined with cost estimates of other expenses associated with the treatment of a calf for BRD (Dubrovsky et al., 2019b). Cost of calfhood BRD was then used to estimate the cost-benefit of implementing 2 specific preventative measures, feeding more milk and vaccination, using different assumptions about BRD cumulative incidence, size of the dairy herd, and bull calf retention scenarios.

\section{MATERIALS AND METHODS}

\section{Study Herds}

The 5 study herds that made up the study cohort were a convenience sample selected based on size, diversity of management practices, record-keeping systems, and owner willingness to participate, and are described in detail in the companion BRD 10K studies (Dubrovsky et al., 2019a,b). The herds enrolled were targeted to include a range of dairy farm herd sizes from California's 3 milk sheds-Northern California (2 dairy farms) and the northern San Joaquin Valley (2 dairy farms), and greater Southern California (1 dairy farm) - and were representative of California dairy farm management systems (Love et al., 2016). The study herds were enrolled between March and August 2015 and unenrolled between June and August 2016. The study was approved by the Institutional Animal Use and Care Committee at the University of California, Davis (Protocol \#17496).

\section{Calf Enrollment and Records}

The study used field data for 11,470 calves on the 5 study farms. Calves were enrolled at birth and followed until weaning, at which point they were censored from the cohort. Calves that were diagnosed and treated for $\mathrm{BRD}$, sold, or died before weaning were assigned the date of those events as their event of interest or censoring date. Treatment records for individual calves were collected using treatment cards affixed to the calf hutches at birth and filled out by the calf treatment crew at the time of BRD treatment, with a total of 2,609 calves treated at least once for BRD. If the dairy farms kept mortality records, the causes of death from the dairy farm records were used to define whether a calf's death was attributed to BRD. Mortality was only examined from birth to weaning and postweaning mortality was not included in the data set.

\section{BRD Morbidity Cost}

A BRD case was defined as a calf diagnosed with BRD that completed a BRD treatment protocol. A second case could only be observed if a calf was treated for BRD previously and likewise for a third. For a calf to be considered a second or third case, the calf must have completed a multi-day treatment protocol for the first case of BRD and have been asymptomatic for at least $2 \mathrm{wk}$. Very few calves were treated for more than 3 cases of BRD, so fourth or fifth cases were excluded from analysis to generate an accurate treatment cost. Study farms were surveyed on what clinical signs were used to identify cases of BRD; all responded that calf caretakers used the clinical signs of nasal discharge, coughing, depression, and pyrexia to identify BRD cases. Medication cost for up to 3 BRD possible cases was calculated based on all the treatment protocols associated with each of the study farms and included a medication cost that was unique for each case. All cost estimates presented in this paper are in US dollars.

A flowchart is presented in Figure 1 to illustrate the relationship of the equations presented in the following section. The equation below represents the medication cost for a given case of BRD per calf $(j=1,2,3)$ without or with $(k=0,1)$ anti-inflammatory medications administered (corticosteroid or the nonsteroidal anti-inflammatory drug flunixin meglumine):

$$
\text { Medication } \operatorname{cost}\left(M_{j k}\right)=\frac{\sum_{i=1}^{n_{j}} T_{i j k} C_{i j k}}{\sum_{i=1}^{n_{j}} C_{i j k}},
$$

where $M_{j k}$ is medication cost for the $j$ th case $(j=1$, $2,3)$, without or with anti-inflammatory medications $(k=0,1)$ summed over all $i$ protocols; $T_{i j k}$ is the cost of $j$ th case with the $i$ th treatment protocol with or without anti-inflammatory medications, and $C_{i j k}$ is the number of calves in the $j$ th case treated with the $i$ th treatment protocol without or with anti-inflammatory medications. The $n_{j}$ notation indicates that for a given case, the number of treatment protocols differed, and 
depending on the drug trade name, there could be up to 32 different protocols, and different cases may have a different set of protocols. The term "protocol" is used to refer to the medications and the number of doses of that medication administered to treat a case of BRD. These protocols were developed by the herd veterinarian and generally involved multiple doses based on the recommendation of the manufacturer (most antibiotics used were not indicated for single dosage use). Some protocols were reserved for calves that calf caretakers felt had more clinical signs or for cases where the calf had already been previously diagnosed and treated for BRD. Dose only refers to a single amount of medication given at a single time point based on the weight of the animal. Antibiotic drug classes used across the farms included phenicol, $\beta$-lactam, fluoroquinolone, tetracycline, macrolide, and aminoglycoside compounds. Some dairy farms did use the same treatment protocols, whereas other treatment protocols were unique to a dairy farm. Dairy farms 1, 2, and 3 routinely used antiinflammatory medications in their protocols, whereas farm 4 never used them and farm 5 only rarely used them. The medication cost $(k=0)$ generated excluded the cost of anti-inflammatory medications but not the protocols that used anti-inflammatory medications. The cost of purchasing the medication was averaged from a sample of 4 representative dairy veterinarians practicing in California's Central Valley. The cost per dose was calculated (with the assumption that the calf was 4 wk of age and weighed $49.9 \mathrm{~kg}$ ) by dividing the cost per bottle of medication by the amount administered based on the concentration $(\mathrm{mg} / \mathrm{kg})$ specified by the manufacturer. The highest cumulative incidence of BRD observed in the BRD $10 \mathrm{~K}$ study was observed at $4 \mathrm{wk}$ of age, which is how the most likely weight was determined for cost per dose; weight was based on birth weight and ADG observations from a study in the same geographic region in the previous $10 \mathrm{yr}$ (Aly et al., 2013). Eleven calves were reported as treated but no medication was recorded on the treatment cards by the calf caretaker and these calves were excluded from all analyses.

The preweaning cost of each BRD case diagnosed and treated was based on the cost of medication, loss

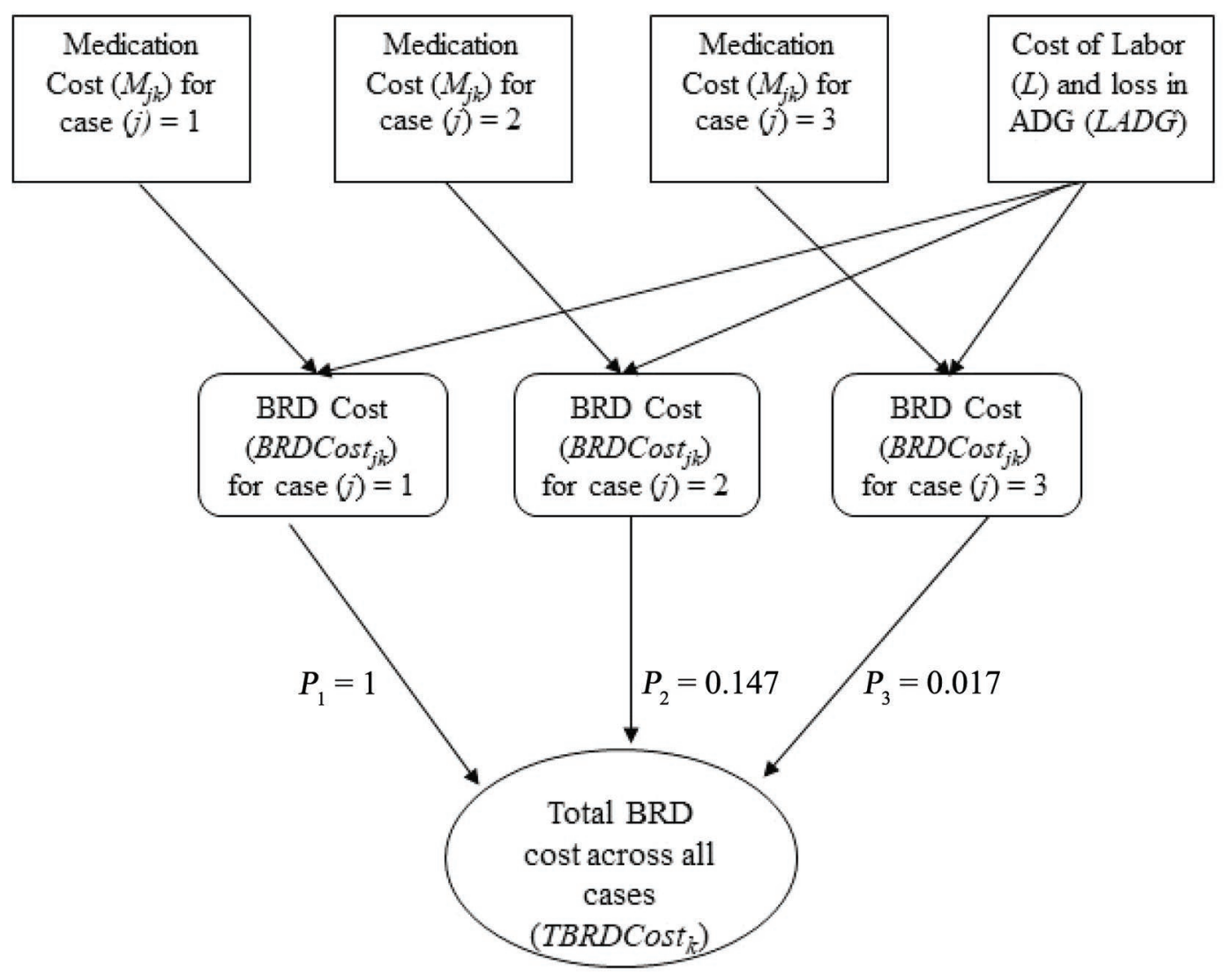

Figure 1. Flowchart illustrating the relationship between Equations [1] (medication cost; $\left.M_{j k}\right),[2]$ (BRD cost; BRDCost ${ }_{j k}$ ), and [3] (total BRD cost; TBRDCost $k_{k}$, where $P_{j}$ is the proportion of calves that had case $j=1,2$, or 3 of bovine respiratory disease (BRD), and $k$ is 0 (without anti-inflammatory medication) or 1 (with anti-inflammatory medication). 
of average daily gain $(L A D G)$, and extra cost of labor (equation [2]). Using the medication cost data, the cost of BRD per case was estimated as follows:

$$
\text { BRDCost }_{j k}=M_{j k}+l+L A D G,
$$

where $B R D$ Cost $_{j k}$ is the BRD cost for case $j$ and $k$ (with or without anti-inflammatory medications), $M_{j k}$ is the medication cost as described above, $l$ is the fixed medication labor cost, and $L A D G$ was the fixed dollar loss in ADG. We calculated $L A D G$ with the assumptions that calves with BRD were utilizing all the additional nutrients ingested (beyond maintenance requirements) for a period of $7 \mathrm{~d}$, to support the inflammatory response, causing no ADG to occur, and was calculated using only the cost of milk, assuming negligible consumption of starter ration. The use of the term "milk" refers to an average of milk replacer, non-saleable, and saleable milk. For $L A D G$, a previous paper estimated that no growth occurred for approximately $7 \mathrm{~d}$ for the severe case of calfhood BRD, which had 3 categories: mild, severe, and chronic (van der Fels-Klerx et al., 2001). The cessation of growth meant that all of the milk fed for the $7 \mathrm{~d}$ when there was no daily gain due to illness was a loss. The mean cost of non-saleable milk, saleable milk, and milk replacer was calculated using the Pennsylvania State's Calf Milk Pasteurization Evaluator spreadsheet (PSCMES; https://animalscience .psu.edu/files/xls/pasteurizer.xls/at_download/file), which includes costs such as energy and labor, as well as fixed investment costs that were independent of herd size, including the purchase and cost of operating a pasteurizer, making the calf milk costs nonlinear as the number of calves on milk increases (Jones et al., 2017). The input cost of milk that was used in the PSCMES was calculated using the descriptive statistics on the calves from the 5 dairy farms in Dubrovsky et al. (2019b), and the input cost of saleable milk was the mean cost for saleable milk during the study period (March 2015 to August 2016). Of the calves enrolled in Dubrovsky et al. (2019b), the mean weaning age was 63 $\mathrm{d}$, the mean number of calves on milk at any given time was 261 calves, and the mean amount of milk fed from birth to weaning was $2.84 \mathrm{~L}$ twice per day. The type of milk fed varied but saleable, non-saleable, and milk replacer were all used by more than one dairy. All saleable and non-saleable milk was assumed to be pasteurized, and replacer was assumed not pasteurized. The price of saleable milk used in PSCMES was calculated for the study period (March 2015 to July 2016) based on USDA's agricultural prices as $\$ 0.36 / \mathrm{kg}(\$ 16.35 / 100$ lb.). Each new or recurring BRD case was assumed to require, on average, an additional hour of labor by calf caretakers for the entire treatment protocol, including time needed to detect or diagnose the new case of BRD as well as providing supportive care, including helping cases to consume their daily milk ration. According to the US Bureau of Labor Statistics, the average wage per hour for farm workers in California in May 2016 (during the data collection period) was $\$ 14.87 / \mathrm{h}$ (US Bureau of Labor Statistics, 2017). Therefore, the estimated treatment protocol labor cost was assumed to be $\$ 14.87 / \mathrm{h}$. The total BRD cost (TBRDCost) across cases is described as follows:

$$
\operatorname{TBRDCost}_{k}=\sum_{j=1}^{3} P_{j} \text { BRDCost }_{j k},
$$

where $P_{j}$ is the proportion of calves treated for a first case of BRD $\left(P_{1}=1\right)$ and diagnosed and treated with a second $\left(P_{2}=0.147\right)$ or third case $\left(P_{3}=0.017\right)$ of BRD.

The total medication $(T M)$ costs, which includes all cases without or with anti-inflammatory medications, were calculated as follows:

$$
T M_{k}=\sum_{j=1}^{3} P_{j} M_{j k}
$$

\section{BRD Incidence by Week of Age}

Incidence of BRD varies by age of calf, which may affect the cost-benefit of preventative measures. The weekly BRD incidence in the calf cohort was calculated for 1 to 9 wk of age using the equation

$$
I_{w}=\frac{C B R D_{w}}{C R I S K_{w}}
$$

where $I_{w}$ is the incidence calculated for calves in the wth week of life, where $w=1,2, \ldots, 9 ; C B R D_{w}$ are calves diagnosed with BRD in the wth week of life; and $C R I S K_{w}$ are the calves at risk of BRD in the wth week of life, which is equal to the total calves alive in wk 1 minus all the calves that were diagnosed with BRD, died, sold, or weaned in $w-1$ wk of life. Repeat cases of BRD in calves were not included in the incidence calculation. All calves sold within $2 \mathrm{~d}$ of birth on the enrolled dairy farms were not included in the weekly BRD incidence calculation.

\section{Mortality Cost}

Mortality Cost Based on Auction Sale Prices. The cost of a BRD mortality was specified using re- 
placement heifer sale prices based on a local auction yard, and estimates from the study dairy farms wherever possible. The cost per calf was represented by the equation

$$
\text { Mortality cost }=F+l+R+\sum_{j=1}^{3} P_{j} M_{j 1}
$$

where $F$ is the cost of the milk fed to the calf before its death. The cost of milk fed before death was assumed to be the average cost of feeding $2.84 \mathrm{~L}$ of milk twice per day (the average amount of milk fed from the cohort of 5 dairy farms) for $33 \mathrm{~d}$, the mean age of mortality for the cohort. The mean cost of milk (\$1.54) was calculated as the mean cost of non-saleable milk (\$0.51), replacer (\$1.88), and saleable milk (\$2.23). The labor cost, $l$, was assumed to be $2 \mathrm{~h}$ for each calf, which included labor to harvest and feed colostrum, treat the calf for BRD, dispose of the dead calf, and clean and quarantine the calf hutch. The cost to replace the dead calf, $R$, was the average cost to purchase a replacement Holstein heifer. Because preweaned calves are not commonly sold, records from the Turlock Livestock Auction (Turlock, CA), which is centrally located in the region of the study dairy farms and has weekly dairy sales, were queried for the study period. The lowest heifer BW category was 128 to $181 \mathrm{~kg}$ (mean $140.38 \mathrm{~kg}$ ), ages 4 to $8 \mathrm{mo}$ old, and sold at $\$ 5.48 / \mathrm{kg}$. The mean price per kilogram was multiplied by $57 \mathrm{~kg}$, the mean weight of heifer calves at $33 \mathrm{~d}$ of age based on the PennState Extension's customized dairy heifer growth chart spreadsheet (https://extension.psu.edu/downloadable/ download/sample/sample_id/951/). The mean price was generated from all weekly dairy sales and not from any special sales or video sales. The average medication cost per calf was the sum of the medication cost over 3 cases, from equation [1], assuming the use of antiinflammatory medications $(k=1)$.

Mortality Cost Based on Variation in Newborn Calf Value. A secondary, more general approach was specified to calculate the cost of a BRD mortality, allowing for variation in the value of a newborn calf ranging from $\$ 100$ to $\$ 300$ in $\$ 50$ increments. The costs incorporated into this mortality estimate included feed, medication, vaccination, housing, and bedding. Remaining costs were itemized into cost of colostrum (\$16.21, labor to harvest and feed the colostrum, assuming $\$ 14.87 / \mathrm{h}$ rate and $1.1 \mathrm{~h}$ of effort), milk fed (\$83.12, based on feeding milk replacer with $28 \%$ protein and $18 \%$ fat valued at $\$ 60 / 22.68-\mathrm{kg}$ bag reconstituted to $15 \%$ solids and fed at a rate of $4.7 \mathrm{~L} / \mathrm{d}$ for $7 \mathrm{~d}$, followed by $6.6 \mathrm{~L} / \mathrm{d}$ through $33 \mathrm{~d}$ of age), starter grain $(22 \% \mathrm{CP}$ valued at $\$ 480 / \mathrm{t}$ and fed at $0.032 \mathrm{~kg} / \mathrm{d}$ for $7 \mathrm{~d}$, and then $0.36 \mathrm{~kg} / \mathrm{d}$ for $26 \mathrm{~d}$ ), and labor by calf caretak- ers (\$8.14 assuming $\$ 14.87 / \mathrm{h}$ rate and $0.5 \mathrm{~h}$ of effort). The following itemized costs were also added based on an author's (MO) unpublished data: medication costs (\$13.55 cost of respiratory protocols) and housing and bedding ( $\$ 15.99$, based on amortization of fixed housing investment costs over 8 yr and 5.2 uses per year). Medication costs were formulated with a treatment protocol commonly used on dairy farms with the costs of the antimicrobials and supportive therapy obtained from an online veterinary pharmaceutical supplier. Vaccination costs were also estimated from an online veterinary pharmaceutical supplier (and adjusted to account for mortality). Housing was assumed to be a fiberglass single-calf hutch with a purchase cost of $\$ 250$ amortized over $8 \mathrm{yr}$. The hutches were assumed to be used by 5.2 calves per year. Bedding was estimated from published extension estimates using shavings or straw. In addition, interest (7\% rate) on feed, housing, and labor (\$0.08) and on investment for each newborn calf was estimated (\$0.57, $\$ 0.85, \$ 1.13, \$ 1.41, \$ 1.70$ for newborn calf values of $\$ 100, \$ 150, \$ 200, \$ 250, \$ 300$, respectively).

\section{Preventative Measures}

Two preventative measures were shown to be associated with a statistically significant $(P<0.05)$ decrease in calfhood BRD in the BRD $10 \mathrm{~K}$ study (Dubrovsky et al., 2019b). Feeding $\geq 4.2 \mathrm{~L} / \mathrm{d}$ of milk versus $3.8 \mathrm{~L} / \mathrm{d}$ or less to calves from birth to $21 \mathrm{~d}$ of age reduced the incidence of BRD by $92 \%$ in calves being fed additional milk during their first $21 \mathrm{~d}$. The same study showed that feeding larger volumes of milk after $21 \mathrm{~d}$ of age was not associated with a reduction in incidence of BRD. The second preventative measure that was shown to be correlated with a $67 \%$ decrease in risk of BRD was the administration of a modified live vaccine against respiratory pathogens during the dam's pregnancy (Dubrovsky et al., 2019b). These 2 preventative measures were used to assess the cost-benefit of several different scenarios, including varying herd sizes, birth rates, and proportion of bulls raised on the dairy farm. The costs of implementing these 2 preventative measures were compared with the short-term benefits associated with decreasing the incidence of BRD.

\section{Herd Size and Management Assumptions}

Two herd sizes were modeled for this cost-benefit analyses, the first was the average 2016 California dairy farm herd size of 1,249 milking cows (CDFA, 2017). The second was the average 2016 US herd size of 223 milking cows (Geiger, 2017). Cumulative incidence rates of $3,5,10,15,20,22.8$, and $25 \%$ were modeled 
for both herd sizes, based on observed field cumulative incidence rates (Dubrovsky et al., 2019b). For each cumulative incidence rate, a total cost of BRD per year was calculated. The mean weaning age was assumed to be 9 wk of age (USDA, 2016). A birth rate (mean number of calves born per year on a given dairy farm) of 1.25 calves per milking cows (not dry cows) was used based on a sample of US dairy farms and was calculated from the mean number of calves born per milking cow per year for the United States (Overton, 2001). Unless otherwise stated, we also assumed that US dairy farms only raised heifer calves and sold all bull calves at birth. It was further assumed that an equal number of heifer and bull calves were born in a year; that is, no use of sexed semen. The term "scenario" hereafter refers to a possible situation and has a specific birth rate, herd size, and bull retention rate in this study. Some scenarios included raising bull calves; in those cases, the percentage of bulls retained was stated.

\section{Herd Scenarios}

Six scenarios were modeled using different herd sizes, birth rates, and proportions of bulls retained. The last 4 herd scenarios explored variations on the average California herd. The third scenario was calculated for a lower than average birth rate of 1.15 calves per milking cow and assumed only heifer calves raised on the dairy farm. The fourth scenario was calculated for a higher than average birth rate of 1.35 calves per milking cow and assumed only heifer calves raised on the dairy farm (which might be observed on a dairy farm utilizing sexed semen and embryo transfer extensively). The fifth scenario was calculated assuming $20 \%$ of all bull calves were retained on the dairy farm (60\% of all calves born per year were raised) and an average birth rate of 1.25 calves per milking cow. The sixth scenario was calculated assuming $40 \%$ of all bull calves were retained ( $70 \%$ of all calves born per year were raised) and an average birth rate of 1.25 calves per milking cow. The birthrate and retained bull calves affect the amount of money saved by implementing 1 of the 2 preventive measures because all pregnant cows were vaccinated regardless of whether they had a bull or heifer calf.

\section{Calculations for BRD Costs}

The number of calves raised on a dairy farm in a given year was calculated as the product of milking herd size times birth rates and the proportion of calves raised. The number of BRD cases on a dairy farm was determined by multiplying the given cumulative incidence rate with the number of calves raised. The total cost of BRD per year was calculated by multiplying the number of calves with BRD by the total cost of BRD calculated from equation [3], assuming use of anti-inflammatory medications in the medication cost.

\section{Calculations for Preventative Measure Implementation}

The costs of the preventative measures were calculated assuming all calves were included in implementation of the preventative measures. For the first preventative measure examined - an increased amount of milk (mean of saleable, non-saleable and milk replacer described under BRD morbidity cost) fed from birth to $21 \mathrm{~d}$ of age - we assumed that calves were fed 3.79 L/d normally and the preventative effect came from increasing the amount of milk fed to $4.26 \mathrm{~L} / \mathrm{d}$ (an extra $0.47 \mathrm{~L} / \mathrm{d}$ of milk), reducing the incidence of BRD by 92\% (Dubrovsky et al., 2019b). All assumptions about processing and cost of saleable milk were the same as the assumptions used for equation [2] for the loss in ADG. The PSCMES, which generates the cost of milk per calf, requires an input of calves on milk, which is determined as [(total calves per year $) /(12 \mathrm{mo})] \times$ months on milk. The months on milk was calculated using $2 \mathrm{mo}$ as an approximation of the $63 \mathrm{~d}$ of age at weaning.

For the cost of administration of the modified live vaccination, all cows that gave birth in a given year were assumed vaccinated (birth rate multiplied by the number of milking cows in the herd) with the total cost of vaccination being the cost per dose per cow, multiplied by the number of cows that calved in $1 \mathrm{yr}$. The vaccination cost per cow was $\$ 3.32$ per dose of vaccine and was calculated from the mean cost of 3 modified live vaccines, which contain BRD pathogen antigens typically administered to pregnant cows and reported previously (Dubrovsky et al., 2019b). Vaccination was assumed to result in a $67 \%$ decrease in BRD incidence based on the BRD 10K study (Dubrovsky et al., 2019b). The number of cows vaccinated annually was calculated by multiplying the number of milking cows in the herd by the birth rate.

All preventative measures resulted in a decrease in the number of calves with BRD and was determined as $(1$ - proportional decrease in BRD) times the number of calves with BRD if no preventative measures were applied at each cumulative incidence condition. The resulting number of calves with BRD was then multiplied by the same cost per calf described in equation [3] to generate a new cost of BRD based on the decrease in cumulative incidence of BRD.

The total amount of money saved or lost by implementation of the preventative measures for all calves on 


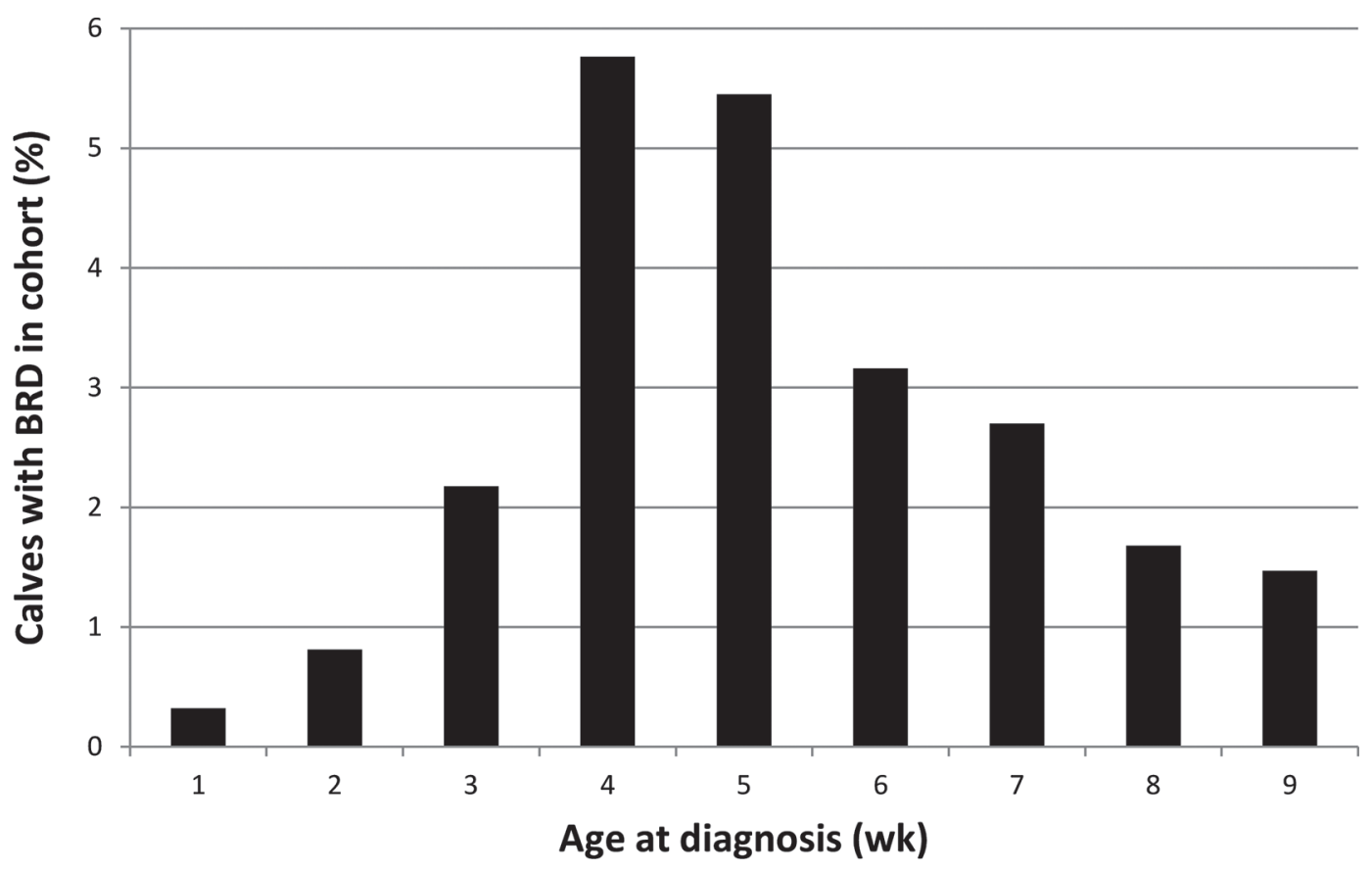

Figure 2. Bovine respiratory disease (BRD) incidence by week of age in a cohort of 11,470 calves. Percentage of calves with BRD was calculated by the number of diagnosed BRD cases each week divided by all calves at risk in a given week.

a dairy farm was calculated by using the cost of BRD without preventative measures, subtracting the cost of reduced $\mathrm{BRD}$ due to a given preventative measure, and then subtracting the cost of a given preventative measure. If the result was positive, then the preventative measures saved money related to the short-term costs associated with calfhood BRD treatment; if negative, the preventative measures resulted in lost income. Longterm implications of calfhood BRD on cow performance and longevity were not included in this analysis.

\section{RESULTS}

\section{Morbidity}

A total of 2,612 first cases of BRD were observed in the cohort of 11,465 calves (22.8\%). The BRD incidence by week of age in the study cohort was highest during wk 4 of life (Figure 2). The cost of BRD per calf is presented in Table 1. A mean of $14.7 \%$ (range: $8.4-19.4 \%$ ) of calves had a second case of BRD after being diagnosed and treated for a case prior. The percentage of calves that had a third case of BRD (out of the total number of calves with a first case of BRD) was $1.7 \%$ (range: $0-2.2 \%$ ). The average number of treatments once a calf was diagnosed with BRD is 1.164. Only 5 calves were treated for a fourth case of $\mathrm{BRD}$, and 1 calf was treated for a fifth case (on a dairy farm that weaned calves later than the study average); hence, these data were not included in the model. Cost of labor was the largest contributor to the overall cost of treating BRD for all cases and accounted for $40 \%$ of costs from equation [3]. The cost of medication accounted for between 37 and $38 \%$ (depending on inclusion of anti-inflammatory drugs) of the cost associated with the treatment of a first case of BRD.

\section{Mortality}

During the study period, 52 calves had deaths attributed to BRD with 3 of the calves being diagnosed but never treated for BRD before death. Thirty-five calves were treated for one case of BRD before death, 11 were treated for BRD twice before death, and 1 each was treated for a third, fourth, and fifth case of BRD before death. The total cost of mortality attributed to BRD was $\$ 395.49$ per calf based on auction sale prices. Assuming variation in newborn calf value, the cost of mortality attributed to BRD was $\$ 243.19, \$ 293.47$, $\$ 343.75, \$ 394.04$, and $\$ 444.32$ for newborn calf values $\$ 100, \$ 150, \$ 200, \$ 250$, and $\$ 300$, respectively. Appendix Table A1 summarizes mortality by dairy farm and case occurrence.

\section{Preventative Measures}

A basic summary of the 6 cost-benefit scenarios and cost per calf for preventative measures is presented in 
Table 1. Cost of treatment (US\$) with and without anti-inflammatory medications (AIM) for treating a calf for bovine respiratory disease $(\mathrm{BRD})^{1}$

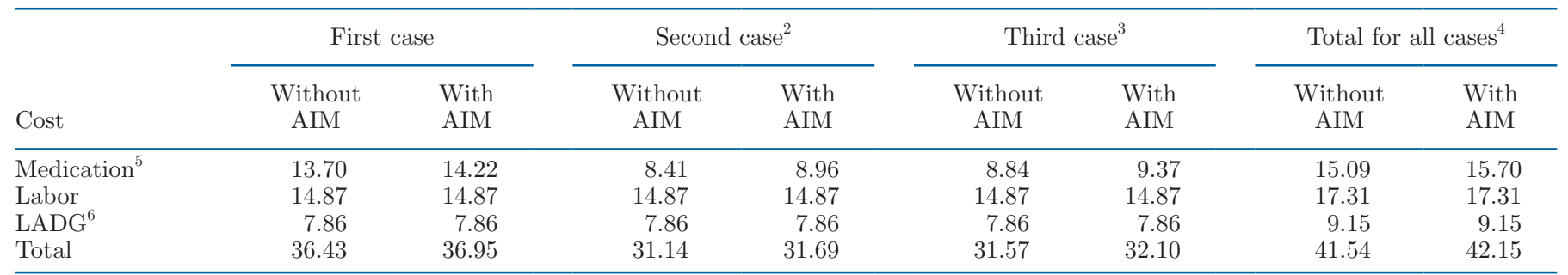

${ }^{1}$ Cost was stratified by recurring BRD cases. The cohort consisted of 2,610 calves diagnosed with BRD from calf population of 11,470 derived from a year-long longitudinal study on the epidemiological characteristics of BRD in calves on California dairies from birth to weaning that took place between March 2015 and August 2016.

${ }^{2}$ Of all calves treated for a first case of BRD, $14.7 \%$ were diagnosed with a second case of BRD.

${ }^{3}$ Of all calves treated for a first case of $\mathrm{BRD}, 1.7 \%$ were diagnosed with a third case of BRD.

${ }^{4}$ The totals were calculated using the sum of the cases and incorporating the proportion of recurrence of second (0.147) and third (0.017) cases. ${ }^{5}$ The cost of medication was generated by calculating the cost per dose of the medication for each of the treatment protocols and then multiplying the average cost of all different treatment protocols by the number of calves that used each given treatment protocol.

${ }^{6}$ Loss in average daily gain (LADG) is the average of replacer $(\$ 13.23)$, non-saleable $(\$ 2.17)$, and saleable $(\$ 8.19)$ milk fed to the calves for $7 \mathrm{~d}$.

Table 2, and the first and second cost-benefit scenarios are presented in Tables 3 and 4 . The first cost-benefit scenario, calculated for an average California herd, is presented in Table 3. The increase in cost when feeding $4.26 \mathrm{~L} / \mathrm{d}$ versus $3.79 \mathrm{~L} / \mathrm{d}$ of milk, using the average of the costs of milk replacer and saleable and non-saleable milk, was $\$ 0.06$ per calf per day. Feeding this increased amount of milk was offset by savings resulting from reduced BRD cumulative incidence at or above $5 \%$ cumulative incidence of BRD, and use of modified live vaccination of dams was financially beneficial at or above $20 \%$ cumulative incidence. The vaccination of the dam with a modified live vaccination cost $\$ 4.64$ per calf assuming no bull calves were raised on the dairy farm. These results suggest that feeding a larger volume of milk to calves would be cost beneficial in terms of reduced BRD incidence for all dairy farms with a cumulative BRD incidence greater than 3.0\% (the lowest rates of $\mathrm{BRD}$ ).

The second cost-benefit scenario was calculated for an average US herd and is presented in Table 4. The increase in cost when feeding $4.26 \mathrm{~L} / \mathrm{d}$ compared with $3.79 \mathrm{~L} / \mathrm{d}$ of milk was $\$ 0.03$ ( $\$ 87.57$ cost of feeding 0.47 $\mathrm{L}$ extra per day $/ 139$ calves $/ 21 \mathrm{~d}=\$ 0.03)$ per calf per day. Feeding an increased amount of milk to calves was cost beneficial at the cumulative incidences of BRD presented in Table 4 and resulted in savings of $\$ 74.15$ to $\$ 1,260.10$, depending on the cumulative incidence. In contrast, use of a modified live vaccination of dams was financially beneficial at or above $20 \%$ cumulative incidence, and was not found to be cost effective when examining the period from birth to weaning for dairy

Table 2. Summary of 6 scenarios for cost-benefit analyses for implementation of 2 preventative measures (increased milk fed or modified live vaccine) on dairies for both an average US and California dairies, different birth rates, proportion of male calves raised, and cost per calf for implementation of preventative measures

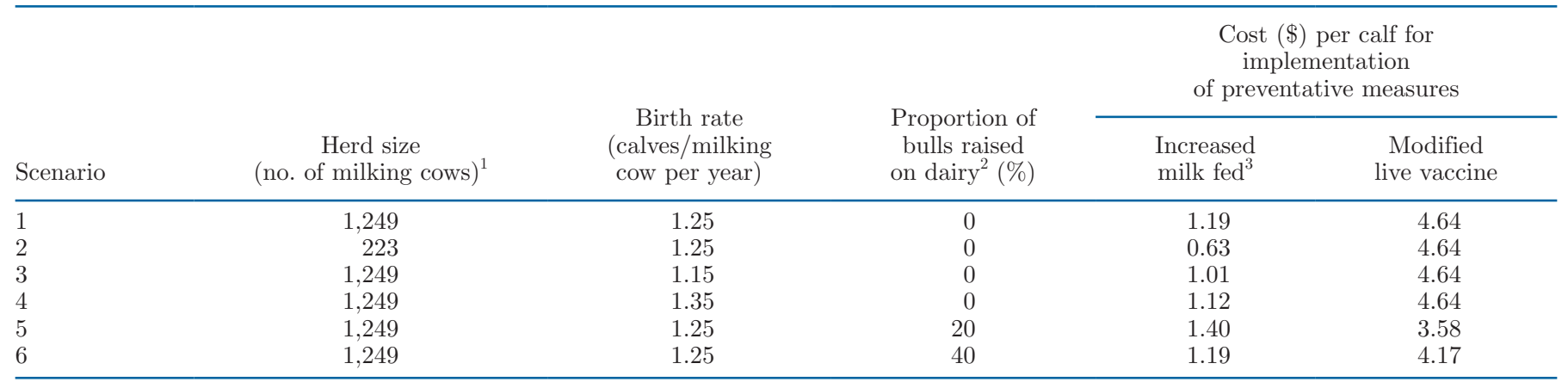

${ }^{1}$ An average California dairy is 1,249 milking cows, whereas an average US dairy is 223 milking cows.

${ }^{2}$ If the proportion of bulls raised is $0 \%$, it was assumed that the dairy was only raising female heifers and selling all male calves within 1 to 2 d of birth.

${ }^{3}$ Additional $0.47 \mathrm{~L}$ of milk (difference between feeding 3.79 and $4.26 \mathrm{~L}$ of milk) for first $21 \mathrm{~d}$ of life for all calves born in a full year. Milk cost was calculated using Pennsylvania State's Calf Milk Pasteurization Evaluator spreadsheet (https://animalscience.psu.edu/files/xls/pasteurizer .xls/at_download/file), which does not scale linearly with number of calves on milk due to fixed input costs. 


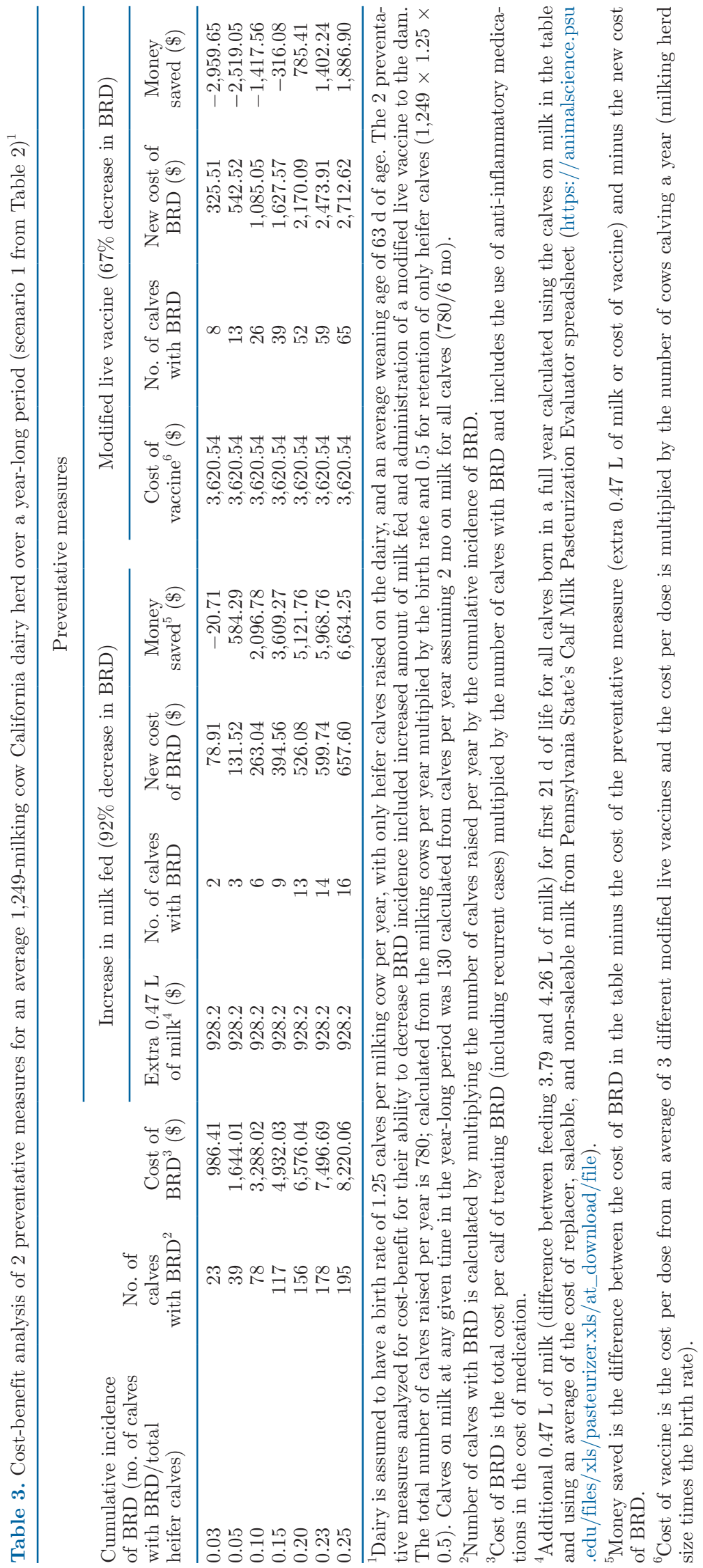




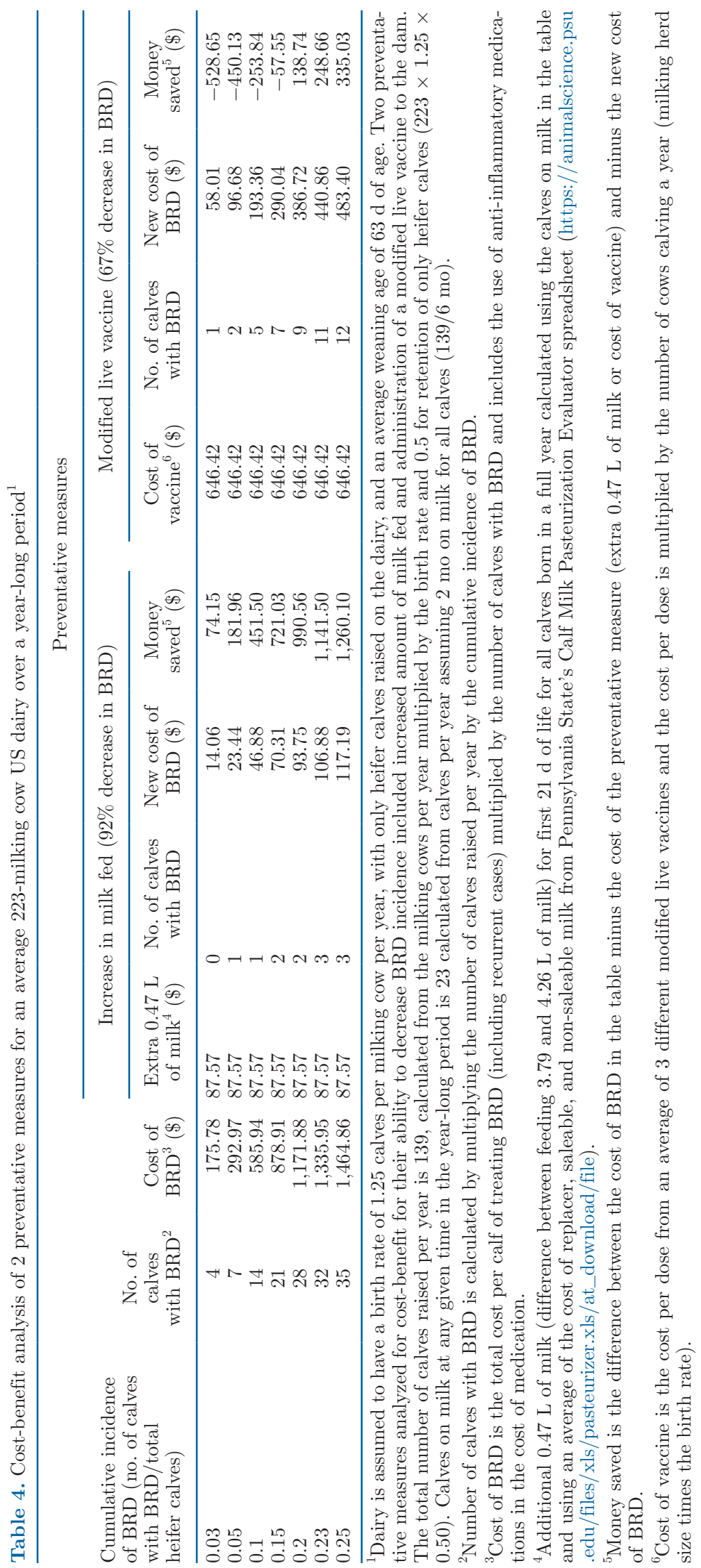


farms with lower risk of BRD, assuming that the benefits were limited only to BRD reduction in the farmraised offspring.

\section{Birth Rate Scenarios}

For the third scenario where the birth rate was dropped to 1.15 calves per cow per year (a decrease of 0.10 calves per cow per year from the average), implementing an increase in milk fed was profitable at or above a $5 \%$ cumulative incidence of BRD. Potential savings from implementing an increase in milk fed per day ranged from $\$ 533.97$ for $5 \%$ cumulative incidence to $\$ 6,106.91$ at $25 \%$ cumulative incidence of BRD.

For an average California dairy farm when the birth rate increases from 1.25 to 1.35 calves per cow per year, the owners stand to profit from implementing an increase in the amount of milk fed at all cumulative incidences of BRD. The potential savings from feeding the extra $0.47 \mathrm{~L}$ of milk ranged from $\$ 36.63$ at a $3 \%$ cumulative incidence of BRD to $\$ 7,229.10$ at a $25 \%$ cumulative incidence of BRD per herd. The results from implementation of increased amounts of milk fed suggest that the majority of dairy farms, except those with the very lowest level of ( $3 \%$ cumulative incidence) of $\mathrm{BRD}$, would benefit from a positive return on their investment.

For both birth rate scenarios (1.15 and 1.35 calves per cow per year), it was only financially beneficial to implement a modified live vaccination for dams with above $20 \%$ cumulative incidence of BRD. The value of the $\mathrm{BRD}$ vaccine was estimated without consideration of the potential benefit to the dam (i.e., only the calf's benefit was examined). Vaccination savings from the 2 scenarios ranged from $\$ 724.84$ for $20 \%$ BRD cumulative incidence (birth rate of 1.15 ) to $\$ 2,042.09$ for $25 \% \mathrm{BRD}$ cumulative incidence (birth rate of 1.35). Because the average cumulative incidence of BRD on dairy farms in California is $22.8 \%$, it is possible that some dairy farms that effectively manage BRD may not economically benefit from changes in disease incidence during the preweaning period by implementing a modified live vaccination protocol. These results likely underestimate the value of vaccination in terms of protecting against lost productivity resulting from calfhood BRD, the benefits of reduced costs associated with treating male calves, and specifically from the overall benefits of immunization against these respective pathogens in the adult dairy herd.

\section{Bull Calf Retention Scenarios}

The last set of scenarios examined included a set percentage of bull calves being retained and raised on an average California dairy farm (as opposed to the selling of all bull calves at birth). The first of these scenarios assumed that $20 \%$ of bull calves born in a year were raised from birth to weaning and were fed, housed, and managed identically to all heifer calves. It was assumed that the bulls retained on the dairy farm had a value similar to the heifer calves. With $20 \%$ retention of bulls, it was economically beneficial to increase milk fed for all cumulative incidence levels of BRD save for the $3 \%$ (lowest) cumulative incidence of $\mathrm{BRD}$. The savings associated with BRD before weaning ranged from $\$ 701.90$ (5\% cumulative incidence of BRD) to $\$ 7,969.61$ ( $25 \%$ cumulative incidence of BRD). The administration of a modified live vaccine to all dams saved money if the cumulative incidence of BRD was at or above $15 \%$, with savings ranging from $\$ 349.05$ (15\% cumulative incidence) to $\$ 2,995.45$ (25\% cumulative incidence).

In the last scenario, where $40 \%$ of bull calves were retained by the dairy farm for raising, savings were even greater with the use of a modified live vaccine. The lowest savings were $\$ 1,009.95$ (at $15 \%$ cumulative incidence of BRD) and increased up to $\$ 4,096.93$ (at $25 \%$ cumulative incidence). Compared with the scenarios in which only heifers were raised on dairy farms, the scenarios where $20 \%$ or $40 \%$ of bulls were raised on the farm showed that use of a modified live vaccine as a preventative measure was more financially beneficial. This was because when bulls are not retained and raised on the dairy, the vaccine that is administered to the herd's pregnant cows only immediately affects the dairy farm through the heifer calves being raised. In scenario 1 , it only saved money to administer a vaccine at a $20 \%$ cumulative incidence of $\mathrm{BRD}$; however, it saved money at $15 \%$ cumulative incidence of BRD with retention of 20 to $40 \%$ of the bulls. The costs associated with ill bull calves are incurred by calf-raising enterprises and represent an externality to the dairy farm.

\section{DISCUSSION}

The purpose of this research was to provide an economic analysis of both cost of treatment and cost of preventative measures for BRD. The current study is unique in that cost of medication was generated from thousands of treatment records, and a cost-benefit analysis of preventative measures for the period from birth to weaning was performed for different types of dairy farms. This study is limited because the long-term economic effect of BRD was not evaluated because it may vary by dairy farm and management practices, and the study calves were not followed up postweaning. However, recent papers reported a decrease in production and increased likelihood of early exit from a herd 
due to calfhood BRD (Warnick et al., 1997; Schaffer et al., 2016).

For both preventative measures examined in this study, there was a cumulative incidence rate threshold above which preventative measures were cost effective. Implementing an increased amount of milk fed improved income in all scenarios with the exception of a dairy farm with $3 \%$ or less cumulative incidence of $\mathrm{BRD}$, and with thousands of dollars in savings at or above a $10 \%$ cumulative incidence of BRD for an average California dairy farm. Other dairy farms outside of California with similar herd sizes and similar costs for preventative measures can expect similar benefits as modeled in this paper. The results per calf that are presented in Table 2 can be used to extrapolate costs for dairy farms of different sizes. Large dairy farms had a greater increase in preventive measure cost per calf when contrasting the 2 quantities of milk fed to calves (3.79 and $4.26 \mathrm{~L} / \mathrm{d}$ ), due to the overhead cost associated with the cost of water for cleaning and energy costs associated with the use of HTST pasteurization of non-saleable milk because these costs are not linear. In contrast, vaccinating pregnant dams with a modified live vaccine was financially beneficial only if the dairy farm had $20 \%$ or cumulative incidence of BRD with savings never amounting to more than $\$ 2,042.09$ at a $25 \%$ cumulative incidence of BRD on an average California dairy farm with a birth rate of 1.35 (data not shown). Examining the $22 \%$ cumulative incidence observed in the cohort of calves used for the generation of the treatment costs, it may not be cost effective for some dairy farms to implement vaccination of dams to prevent BRD in calves if consideration of the costs and benefits are limited to the period between birth and weaning. However, this study only considered BRD reduction up to weaning. Calves experiencing BRD in the first $120 \mathrm{~d}$ of their life may experience long-term deleterious effects on their health, and other likely benefits of vaccination for the adult herd were not accounted for in the current study (Schaffer et al., 2016).

The only scenarios in which vaccination became more economical were those involving retention of bull calves for on-site rearing. These savings occurred because all pregnant cows are commonly vaccinated, because the sex of the calf is not typically known before calving. By retaining bull calves, the administration of a vaccine paid off in the decrease in cumulative incidence of BRD as the ratio of calves kept to cows vaccinated increased, which diluted the additional cost of vaccination over a larger number of calves being raised. Based on the results of the cost-benefit scenarios, an increase in the amount of milk fed is a preventive measure that dairy farms should consider implementing, whereas vaccination of dams was only cost beneficial (examining costs associated with calves from birth to weaning) on dairy farms with average or above average cumulative incidence rates of $\mathrm{BRD}$. However, the economic benefits from vaccination are likely to be underestimated in the current analyses as we did not factor in long-term health.

To broadly estimate the cost of BRD from Tables 3 or 4 from any herd size (HS) at any cumulative incidence of BRD (CI), 3 equations were developed under the assumptions in Tables 3 and 4: BRD cost without any treatment [BRDCost(CI, HS)], BRD cost with extra $0.47 \mathrm{~L}$ of milk [BRDCostMilk(CI, HS)], and $\mathrm{BRD}$ cost with vaccination [BRDCostVac(CI, HS)] for any CI and any HS. The 3 equations are as follows: $\mathrm{BRDCost}(\mathrm{CI}, \mathrm{HS})=(0.006737+26.1767 \times \mathrm{CI}) \times \mathrm{HS}$; BRDCostMilk $(\mathrm{CI}, \mathrm{HS})=(0.0005396+2.0942 \times \mathrm{CI}) \times$ $\mathrm{HS}$; and BRDCostVac $(\mathrm{CI}, \mathrm{HS})=(0.002234+8.6382 \times$ CI) $\times$ HS.

The reader should use caution when using these equations because these are only relative costs under the assumptions presented in this paper and other information would provide a more accurate cost estimate. These 3 equations can roughly reproduce columns 3 (base cost of BRD), 6 (new cost of BRD with increase in milk fed), and 10 (new cost of BRD with vaccine) in Tables 3 or 4 with any HS or CI within a reasonable range. The coefficients from the equations above were derived using the results in Table 4 and dividing columns 3, 6, and 10 by 223 (which is the HS in Table 4 ), which were then regressed on column 1 or the CI.

Previous studies (van der Fels-Klerx et al., 2001) focused on developing models that can be used by individual dairy producers (who enter their own costs), whereas this study used individual treatment records from 2,615 calves diagnosed with BRD to estimate an average cost of treatment. Use of treatment records from 5 dairy farms generated an average cost based on 32 combinations of individual antibiotics (including 12 different combinations of different classes of antibiotics) as part of different treatment protocols. The limitation of using the treatment data from the cohort is that results of this paper are heavily dependent on the assumptions made in the BRD 10K longitudinal study from which this cohort is derived. The treatment data also provided records on recurrent treatments of calves for $\mathrm{BRD}$, which were incorporated in the BRD cost and that was not considered in previous studies. One study incorporated the number of doses of medication given for a single case of BRD but no option for incorporating cost of treating recurrent cases of BRD (van der Fels-Klerx et al., 2001). Over $14.7 \%$ of calves diagnosed with BRD were recorded as having a second case before weaning and required a second round of treatment. The proportion of calves with second BRD cases was similar 
to but slightly higher than that of a 2014 study that reported 9.3 and $4.1 \%$ retreatment rates for enrofloxacin and tulathromycin treatment protocols, respectively (Heins et al., 2014). The high proportion of recurrent BRD cases suggests that previous studies may have underestimated the cost of BRD.

One limitation of our study was that classification of BRD was dichotomized as the calf being a case or not, whereas previous studies modeled degrees or severity of BRD. A Netherlands study differentiated cases of BRD as mild, severe, or chronic (van der Fels-Klerx et al., 2001). The difficulty with these categorizations of BRD was that calves were not sorted into these classes using definite diagnostic methods such as ultrasound; rather, observation and subjective assessment of clinical signs were used to categorize the severity of BRD. Our study did not seek to define classes of BRD disease because of the potentially subjective nature of observation of clinical signs (Love et al., 2014). Future studies would benefit from the use of objective diagnostic tests such as ultrasound.

Calves that died were either potentially not treated soon enough after displaying clinical signs (calves that died within a day or two of the initial treatment) or the calves' conditions deteriorated rapidly that treatments were ineffective at preventing permanent damage to their respiratory tracts. In our study, treatment records revealed that $67 \%$ of all calves (35 of 52 ) who died due to BRD had only one treatment protocol administered before death, suggesting the possibility of delays in identifying BRD early enough for treatments to be effective. Another study where calves were followed through weaning reported a subjective increase in BRD incidence after weaning at 9 wk of age (Schaffer et al., 2016).

Unlike other studies that incorporated mortality cost into total cost of BRD, we chose to separate out the cost of treating BRD and the cost associated with a case of BRD-attributed mortality (Kaneene and Hurd, 1990; van der Fels-Klerx et al., 2001). However, similar to previous studies, the mortality cost estimates presented in this paper included costs that were incurred and cannot be recovered such as milk fed, and the cost of replacing the dead calf. For the cost of purchasing a calf to replace a BRD mortality, 2 approaches were presented, which resulted in a $\$ 395$ cost based on the study dairy farms or a general cost that varied by newborn calf value and included interest on investment, which ranged from $\$ 243$ to $\$ 444$. A study in the Netherlands that modeled calfhood BRD costs assumed that a replacement heifer would be a springer and estimated the cost (adjusted for inflation) at $\$ 816.08$ in 2001 (van der Fels-Klerx et al., 2001), which may suggest that the cost of calf mortality attributable to BRD has in- creased over the years potentially due to an increase in prices of replacement animals or, alternatively, due to regional differences.

Cost of treating BRD (including use of anti-inflammatory medications and recurrent cases of BRD), $\$ 42.15$ per calf, was high compared with the costs presented in previous papers that focused on calfhood BRD and included all the cost variables (Kaneene and Hurd, 1990; van der Fels-Klerx et al., 2001). The cost calculated in the Netherlands in 2001, adjusting for inflation, was a median of $\$ 37.17$, with a low of $\$ 21.92$ (for a mild case of BRD) and a high of $\$ 68.03$. At first glance, this $\$ 37.17$ may be similar to the $\$ 42.15$ calculated in our model; however, the Netherlands' cost estimates included several additional costs such as the cost of future decrease in production with variables representing the costs associated with decreased growth and fertility, purchasing of replacement heifers, and the decrease in milk production. Although the actual numbers appear similar, the Netherlands study included many additional factors associated with postweaning lifetime production costs that were not factored into the cost calculated for the current study.

The only previous model developed in the United States was in the 1990s, utilizing data from USDA's NAHMS study, and had the cost of BRD stratified by herd sizes with a range of $\$ 19.59$ to $\$ 49.98$ with an average of $\$ 27.57$ per calf per year (Kaneene and Hurd, 1990). The USDA model, like the Netherlands model, included more variables than the model presented in this paper and included the cost of preventative measures, as well as costs associated with calves that died or were culled due to BRD. A major difference from the previous 2 studies is that the current study utilized average costs for the model inputs. Average costs were chosen because there is considerable variation in presentation and treatment of $\mathrm{BRD}$, involving numerous factors, and trying to present all of the different possible scenarios and treatment protocols individually would have been overly complicated. The goal of the calculations in this study was to provide producers and researchers a general estimate of what a producer might expect to spend when treating BRD in calves, as well as the economic impact of BRD.

The scenarios presented in the current study illustrate both the high cost of BRD and the financial benefits of instituting preventative measures. The only scenarios where it was not financially beneficial to implement preventive measures all had lower than the $22 \%$ cumulative incidence of BRD observed in Dubrovsky et al. (2019b), the most recent longitudinal study on BRD. The estimated total cost of BRD indicates that the cost of treating BRD is higher now than 10 to $20 \mathrm{yr}$ ago, and calfhood BRD continues to have a substantial 
economic impact. Calfhood BRD remains a challenge to the dairy industry, and preventative measures can be an effective tool for minimizing the incidence and improving calf welfare while decreasing both the shortterm and long-term costs of BRD for dairy producers. Our study findings provide producers and veterinarians with an estimate of the current costs associated with treating preweaned calves with $\mathrm{BRD}$, how these costs vary by herd structure, and whether implementation of these preventative measures against BRD in preweaned calves is beneficial

\section{ACKNOWLEDGMENTS}

The authors thank the dairy producers, calf caretakers, and veterinary practitioners for their participation. The current study was funded by competitive grant \#1753 "Risk assessment, welfare analysis, and extension education for dairy calf respiratory disease management in California," Sharif Aly provided by the University of California Division of Agriculture and Natural Resources, the USDA National Institute of Food and Agriculture (Washington, DC), Animal Health project CALV-AH-360 and the USDA "Integrated Program for Reducing Bovine Respiratory Disease Complex (BRDC) in Beef and Dairy Cattle."

\section{REFERENCES}

Aly, S. S., P. Pithua, J. D. Champagne, and D. M. Haines. 2013. A randomized controlled trial on preweaning morbidity, growth and mortality in Holstein heifers fed a lacteal-derived colostrum replacer or pooled maternal colostrum. BMC Vet. Res. 9:168.

CDFA (California Department of Food and Agriculture). 2017. California Dairy Statistics Annual: 2016 Annual Data. https://www .cdfa.ca.gov/dairy/pdf/Annual/2016/2016_Statistics_Annual.pdf.

Dubrovsky, S. A., A. L. Van Eenennaam, B. M. Karle, P. V. Rossitto, T. W. Lehenbauer, and S. S. Aly. 2019a. Bovine respiratory disease (BRD) cause-specific and overall mortality in preweaned calves on California dairies: The BRD 10K study. J. Dairy Sci. 102:7320-7328.

Dubrovsky, S. A., A. L. Van Eenennaam, B. M. Karle, P. V. Rossitto, T. W. Lehenbauer, and S. S. Aly. 2019b. Epidemiology of bovine respiratory disease (BRD) in preweaned calves on California dairies: The BRD 10K study. J. Dairy Sci. 102:7306-7319.

Geiger, C. 2017. Dairy farm exits outpaced 10-year trend. Hoard's Dairyman Intel Vol. February 27, 2017. http://hoards.com/article -20506-Dairy-farm-exits-outpaced-10-year-trend.html.

Heins, B. D., D. V. Nydam, A. R. Woolums, R. D. Berghaus, and M. W. Overton. 2014. Comparative efficacy of enrofloxacin and tulathromycin for treatment of preweaning respiratory disease in dairy heifers. J. Dairy Sci. 97:372-382.
Jones, C., A. J. Heinrichs, B. James, and C. Scott. 2017. Penn State Extension Calf Milk Pasteurization Evaluator Spreadsheet. https: //animalscience.psu.edu/files/xls/pasteurizer.xls/at_download/ file.

Kaneene, J. B., and H. S. Hurd. 1990. The national animal health monitoring-system in Michigan. 3. Cost estimates of selected dairy-cattle diseases. Prev. Vet. Med. 8:127-140.

Love, W. J., T. Lehenbauer, P. H. Kass, A. L. Van Eenennaam, and S. S. Aly. 2014. Development of a novel clinical scoring system for on-farm diagnosis of bovine respiratory disease in pre-weaned dairy calves. PeerJ 2:e238.

Love, W. J., T. W. Lehenbauer, B. M. Karle, L. E. Hulbert, R. J. Anderson, A. L. Van Eenennaam, T. B. Farver, and S. S. Aly. 2016. Survey of management practices related to bovine respiratory disease in preweaned calves on California dairies. J. Dairy Sci. 99:1483-1494.

Overton, M. 2001. Stochastic modeling of different approaches to dairy cattle reproductive management. J. Dairy Sci. 84(Suppl. 1):268 (Abstr.)

Schaffer, A. P., R. L. Larson, N. Cernicchiaro, G. A. Hanzlicek, S. J. Bartle, and D. U. Thomson. 2016. The association between calfhood bovine respiratory disease complex and subsequent departure from the herd, milk production, and reproduction in dairy cattle. J. Am. Vet. Med. Assoc. 248:1157-1164.

Stanton, A. L., D. F. Kelton, S. J. LeBlanc, J. Wormuth, and K. E. Leslie. 2012. The effect of respiratory disease and a preventative antibiotic treatment on growth, survival, age at first calving, and milk production of dairy heifers. J. Dairy Sci. 95:4950-4960.

US Bureau of Labor Statistics. 2017. Occupational employment and wages, May 2016 in 45-2093 farmworkers, farm, ranch, and aquacultural animals. United States Department of Labor, Washington, DC. https://www.bls.gov/oes/current/oes452093.htm.

USDA. 2010. Dairy 2017, Part V: Changes in dairy cattle health and management practices in the United States, 1996-2007. Pages 1-91. Vol. 519.0709. USDA Animal and Plant Health Inspection Service, Veterinary Services, Center for Animal Epidemiology and Health (APHIS:VS, CEAH), Fort Collins, CO.

USDA. 2012. Dairy Heifer Raiser, 2011. APHIS-NAHMS, ed. USDA Animal and Plant Health Inspection Service (APHIS), Fort Collins, CO.

USDA. 2016. Dairy 2014: Dairy cattle management practices in the United States. APHIS-NAHMS, ed. USDA Animal and Plant Health Inspection Service (APHIS), Fort Collins, CO.

van der Fels-Klerx, H. J., J. T. Sorensen, A. W. Jalvingh, and R. B. M. Huirne. 2001. An economic model to calculate farm-specific losses due to bovine respiratory disease in dairy heifers. Prev. Vet. Med. 51:75-94.

Warnick, L. D., H. N. Erb, and M. E. White. 1997. The relationship of calfhood morbidity with survival after calving in 25 New York Holstein herds. Prev. Vet. Med. 31:263-273.

\section{ORCIDS}

A. L. Van Eenennaam ๑ https://orcid.org/0000-0003-1562-162X

S. S. Aly ๑ https://orcid.org/0000-0003-0330-5013

M. W. Overton (๑ https://orcid.org/0000-0002-5168-6319

T. W. Lehenbauer (i) https://orcid.org/0000-0003-0896-3939

J. G. Fadel ๑ https://orcid.org/0000-0002-3512-5036 


\section{APPENDIX}

Table A1. Tally of number of cases of bovine respiratory disease (BRD) morbidity and mortality broken down by dairy ${ }^{1}$

\begin{tabular}{|c|c|c|c|}
\hline Dairy & $\begin{array}{l}\text { BRD case } \\
\text { occurrence }\end{array}$ & $\begin{array}{l}\text { Total no. } \\
\text { of calves }\end{array}$ & $\begin{array}{l}\text { BRD } \\
\text { deaths }\end{array}$ \\
\hline \multirow[t]{4}{*}{1} & 1 & 2,048 & 17 \\
\hline & 2 & 397 & 8 \\
\hline & 3 & 46 & 0 \\
\hline & 4 & 2 & 0 \\
\hline \multirow[t]{4}{*}{2} & 1 & 190 & 0 \\
\hline & 2 & 17 & 0 \\
\hline & 3 & 3 & 0 \\
\hline & 4 & 1 & 0 \\
\hline \multirow[t]{4}{*}{3} & 1 & 178 & 14 \\
\hline & 2 & 29 & 0 \\
\hline & 3 & 5 & 0 \\
\hline & Not treated & & 1 \\
\hline \multirow[t]{6}{*}{4} & 1 & 155 & 2 \\
\hline & 2 & 13 & 2 \\
\hline & 3 & 3 & 1 \\
\hline & 4 & 2 & 1 \\
\hline & 5 & 1 & 1 \\
\hline & Not treated & & 1 \\
\hline \multirow[t]{3}{*}{5} & 1 & 39 & 2 \\
\hline & 2 & 8 & 1 \\
\hline & Not treated & & 1 \\
\hline Totals & & 2,610 & 52 \\
\hline
\end{tabular}

